\title{
The Impact of Diabetes on Acute Post-Infectious Glomerulonephritis in Rats: An Experimental Study with Literature Review
}

\author{
Hala Kfoury ${ }^{1 *}$, Abdulkareem Alsuwaida ${ }^{2}$, Mohammed Alghonaim², Sufia Husain ${ }^{1}$, Tariq Aljuhani ${ }^{1}$, \\ Hanan H Hagar ${ }^{3}$, Safia Moussa ${ }^{4}$ and Ibrahim Ginawi ${ }^{4}$
}

${ }^{1}$ Department of Pathology, College of Medicine, King Saud University, Saudi Arabia

${ }^{2}$ Department of Medicine, College of Medicine, King Saud University, Saudi Arabia

${ }^{3}$ Pharmacology Department, College of Medicine, King Saud University, Saudi Arabia

${ }^{4}$ College of Medicine, Hail University, Saudi Arabia

${ }^{*}$ Corresponding author: Dr. Hala Kfoury, Department of Pathology and Laboratory Medicine (32), PO Box 2925 College of Medicine, King Saud University, Riyadh-11461, Saudi Arabia, Tel: +966-504127781; E-mail: halakfoury@hotmail.com

Received date: October 24, 2017; Accepted date: November 21, 2017, Published date: November 24, 2017

Citation: Kfoury H, Alsuwaida A, Alghonaim M, Husain S, Aljuhani T, et al. (2017) The Impact of Diabetes on Acute Post-Infectious Glomerulonephritis in Rats: An Experimental Study with Literature Review. J Clin Exp Nephrol Vol.2 No.4:50.

Copyright: (c) 2017 Kfoury H, et al. This is an open-access article distributed under the terms of the Creative Commons Attribution License, which permits unrestricted use, distribution, and reproduction in any medium, provided the original author and source are credited.

\section{Abstract}

Background: The concurrence of diabetes and nephritogenic infections may have a tremendous impact on the kidney. The approach to the two concurrent diseases is based on histological, immunofluorescence findings and most importantly on ultrastructural features. In this experiment our aim was to compare the pattern of injury seen in the kidney in animals with induced post-infectious glomerulonephritis with and without associated diabetes.

Methods: We divided 48 Wistar species rats into four groups comprising twelve rats each. Group I is the normal control, group II includes the rats with induced diabetes, group III are the rats with acute post-infectious glomerulonephritis (APIG), while group IV includes the rats with induced diabetes and APIG. Streptozocin was used to induce diabetes in groups II and IV while neutral phosphatase toxin (NPTase) of Staphylococcus strain was used in groups III and IV. Groups III and IV were sacrificed three and ten days after infection. Routine, immunofluorescence and ultrastructural stains and studies were performed on the kidneys of all rats. Serum urea and creatinine and urinary protein were estimated in all groups.

Results: Out of the forty-eight rats initially included in the study, only twenty-four survived the experiment: three in Group I, five in Group II, five in Group III and eleven in Group IV. The most relevant and distinguishing findings in cases of APIG with associated diabetes are the earlier and more prominent infiltration by polymorphonuclear cells, the stronger positivity for IgG and the earlier and more extensive presence of dense deposits which were confirmed by the electron microscopy study.

Conclusions: This experiment ascertains the adverse effects of diabetes in cases of acute post-infectious glomerulonephritis, in terms of onset and severity of the disease. The necessity of a better control of the glucose level may not only decrease the incidence of acute postinfectious glomerulonephritis but may also contribute to the development of a milder form of the disease.

Keywords: Diabetes; Rats; Acute post-infectious glomerulonephritis (APIG)

\section{Introduction}

Diabetic nephropathy (DN) is a well-characterized microvascular complication of diabetes (DM). Approximately $20 \%-40 \%$ of individuals with DM will eventually develop type 2 diabetic nephropathy (T2DN). However, individuals with DM may also develop renal disease due to other causes.

An individual with DM may require renal biopsy to determine the precise etiology of renal disease. Furthermore, renal biopsy provides invaluable information about the severity of glomerular and tubulointerstitial pathology in the kidney.

Acute post-infectious glomerulonephritis (APIGN) is an immune-mediated form of glomerulonephritis that most commonly affects children although it may occur in adults of all age groups. Classically, APIGN develops following streptococcal upper respiratory tract or skin infections [1,2]. More recently, other organisms, particularly Staphylococcus and Gram-negative bacteria, have been linked to APIGN in adults and elderly patients [3-5]. In the developed countries, Staphylococcus has become three times more common than Streptococcus as the responsible infectious agent for APIGN in the elderly population [3]. A less frequent pattern that resembles membranoproliferative glomerulonephritis type 1 by LM with glomerular deposits of IgM and C3 or IgG and C3 occurs in patients with ventriculo-vascular shunt infection by Staphylococcus epidermidis has also been described $[1,2]$. The 
classic glomerular pattern of injury associated with staphylococcal infection, including diffuse endocapillary proliferative and exudative glomerulonephritis on light microscopy (LM), glomerular immune deposits that stain for either IgG and C3 or C3 only on immunofluorescence (IF), and 'humpshaped' subepithelial deposits or at an early stage the presence of subendothelial or mesangial dense deposits on electron microscopy (EM) [1,2].

The aim of this study is to identify a category of clinical and histological findings of high accuracy, sensitivity and specificity which may help in understanding APIG in cases of diabetic nephropathy and also to assess the findings in cases of APIG. Furthermore, we tried to outline the pathogenetic mechanisms in each category and to study the possibility of a different pathological spectrum of APIG in rats with diabetes in comparison to APIG in rats without diabetes.

\section{Materials and Methods}

We divided 48 Wistar rats into four groups of twelve rats each. Goup I is the normal control, group II includes the rats with induced diabetes, group III are the rats with APIG, while the group IV are the rats with induced in a two-step measure of diabetes than acute infection by NPTase toxin of Staphylococcus aureus. Streptozotocin or Streptozocin or Izostazin or Zanosar (STZ) is an antineoplastic agent related to the nitrosureas which is used as a chemotherapy agent in many types of cancers. Streptozotocin sterile powders are provided and prepared as a chemotherapy agent. Each vial of sterilized Streptozotocin powder contains 1 gram of Streptozotocin active ingredient with the chemical name, 2-Deoxy-2-[[(methylnitrosoamino)carbonyl] amino]-D-glucopyranose and $200 \mathrm{mg}$ of citric acid. Streptozotocin is available for intravenous use as a dry-frozen, pale yellow, sterilized product. The dissolution of the Streptozotocin is performed according to the vials guidelines. This material is prepared in 1-gr vials and kept in cold store at $4^{\circ} \mathrm{C}$ and kept in the dark. Group II and IV Wistar rats weighting 250-300 g (75-90 days old) are used for inducing diabetes. The animals are injected by streptozotocin at the dose of $60 \mathrm{mg} / \mathrm{kg}$ of the body weight intravenously. Streptozotocin induces diabetes within 3 days through the destruction of the beta cells of the pancreas. All groups are kept in four cages under feeding and metabolism control. The food and water consumption is similar in all four groups.

Group III and IV are injected with Recombinant 30KDa neutral phosphatase (NPTase) of Staphylococcus strain to induce acute post-infectious glomerulonephritis after dilution with the buffer which includes $0.2 \mu \mathrm{m}$ sterile filtered $20 \mathrm{mM}$ Tris- $\mathrm{HCl}, 0.5 \mathrm{M}$ $\mathrm{NaCl}, \mathrm{Ph} 8.0,20 \%$ glycerol using a concentration of $0.1-5 \mathrm{mg} / \mathrm{ml}$.

Groups III and IV are sacrificed three and ten days after infection while the other groups are sacrificed at ten days postinduction of the infection. Routine, immunofluorescence and ultrastructural stains and studies are performed on the kidneys of all rats. Serum urea and creatinine and urinary protein are estimated in all groups.

The experiment was conducted over a period of 12 weeks. Following initiation of induction of the above mentioned disease conditions, blood and urine samples are obtained from each rat every week and submitted for clinical laboratory tests. The blood samples are tested for serum creatinine and blood urea nitrogen. The urine samples are tested for proteinuria. After 12 weeks the animals are sacrificed and the organs (kidneys) harvested. The organs will be submitted to the histopathology section. From every kidney 5 stained slides are obtained (Hematoxylin and eosin stain, Periodic acid-Schiff stain, Jones silver stain and Masson trichrome stain).The tissues and slides are prepared following optimized and standardized protocols. The tissues are also submitted for immunofluorescence and electron microscopy evaluation.

All the light and immunofluorescence, and immunofluorescence microscopy slides and electron microscopy photomicrographs thus obtained are assessed by two trained nephropathologists. Each group is assessed separately including the dual pathology cases. The histological findings are correlated, validated and subjected to various statistical evaluations.

\section{Results}

Out of the forty-eight rats initially included in the study, only twenty-four survived the twelve weeks experiment: three in Group I, five in Group II, and five in Group III and eleven in Group IV. The most relevant and distinguishing findings in cases of APIG with associated diabetes are the earlier and more prominent infiltration by polymorphonuclear cells as well as the mesangial matrix expansion and the thickening of the glomerular basement membranes(GBM) in both groups sacrificed at three and ten days (Table 1), as well as the stronger positivity for mesangial and focal capillary IgG positivity the earlier and more extensive presence of dense deposits which were confirmed by the electron microscopy study.

Table 1: Glomerular findings in rats with and without diabetes at three and ten days post induction of acute infection.

\begin{tabular}{|c|c|c|c|c|}
\hline \multirow{2}{*}{ P-value } & \multirow{2}{*}{$\begin{array}{l}\text { Mesangi } \\
\text { al Matrix }\end{array}$} & Membrane & \multirow{2}{*}{$\begin{array}{l}\text { Leukocyte } \\
\mathrm{s} \\
\text { infiltration }\end{array}$} & \multirow{2}{*}{ Glomeruli } \\
\hline & & Thickening & & \\
\hline & + & + & ++ & $\begin{array}{l}\text { Diabetes }+ \text { Post } \\
\text { infection (10 days) } \\
\text { ( } n=5 \text { rats) }\end{array}$ \\
\hline \multirow[t]{3}{*}{0.018} & \multirow{2}{*}{-} & \multirow{2}{*}{ - } & \multirow{2}{*}{+} & Post infection \\
\hline & & & & (10 days) ( $n=3$ rats) \\
\hline & + & + & + & $\begin{array}{l}\text { Diabetes }+ \text { Post } \\
\text { infection (3 days) } \\
\text { ( } n=6 \text { rats) }\end{array}$ \\
\hline \multirow[t]{2}{*}{0.036} & \multirow{2}{*}{-} & \multirow{2}{*}{ - } & \multirow{2}{*}{$+1-$} & Post infection \\
\hline & & & & ( 3 days) $(n=2$ rats) \\
\hline
\end{tabular}

By routine microscopy, there was no significant endocapillary proliferation, fibrinoid necrosis, crescents formation, thrombosis, double contour formation or wire loop noted in any of the glomeruli of the four groups. The leukocytes infiltration, the thickening of the membranes and the mesangial matrix expansion showed statistical difference in the group of diabetes 
and concurrent post-infectious glomerulonephritis in comparison to the group with post-infectious glomerulonephritis, mainly when the rats were sacrificed ten days post induction of the infection. In addition, none of the groups showed any acute tubular necrosis or interstitial hemorrhage nor tubular atrophy or interstitial fibrosis.

Table 2: Tubulo-interstitial findings in rats with and without diabetes at three and ten days post induction of acute infection.

\begin{tabular}{|c|c|c|c|c|c|c|}
\hline Tubulo - interstitium & $\begin{array}{l}\text { Interstitial } \\
\text { Inflammation }\end{array}$ & Tubulitis & $\begin{array}{l}\text { Tubular } \\
\text { Atrophy }\end{array}$ & Fibrosis & $\begin{array}{l}\text { Peritubular } \\
\text { Capillarities }\end{array}$ & P-value \\
\hline $\begin{array}{l}\text { Diabetes + Post infection } \\
\text { (10 days) ( } n=5 \text { rats) }\end{array}$ & & - & - & - & & \\
\hline $\begin{array}{l}\text { Post infection } \\
\text { (10 days) ( } n=3 \text { rats) }\end{array}$ & & + & - & - & & 0.018 \\
\hline $\begin{array}{l}\text { Diabetes + Post infection } \\
\text { ( } 3 \text { days) ( } n=6 \text { rats) }\end{array}$ & $=+$ LYM PMN & & & & & \\
\hline $\begin{array}{l}\text { Post infection } \\
\text { ( } 3 \text { days) ( } n=2 \text { rats) }\end{array}$ & & & & & & 0.036 \\
\hline
\end{tabular}

The interstitial inflammation and the peritubular capillaritis were more prominent in group IV especially in the rats sacrificed at ten days post induction of the infection, (Tables 1 and 2; Figures 1 and 2). The arteries and arterioles did not show significant pathology in any of the groups.

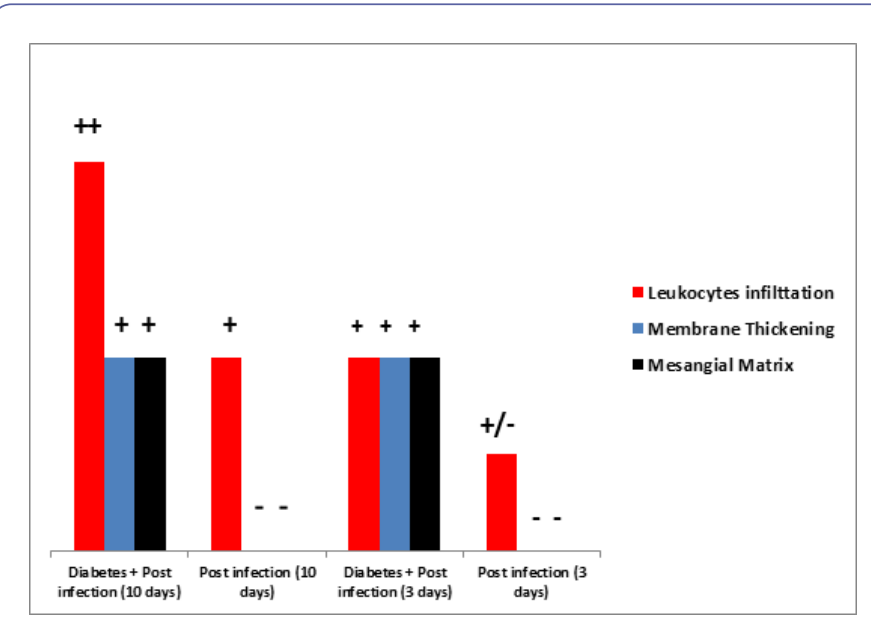

Figure 1: Glomerular changes in post-infectious cases with and without diabetes.

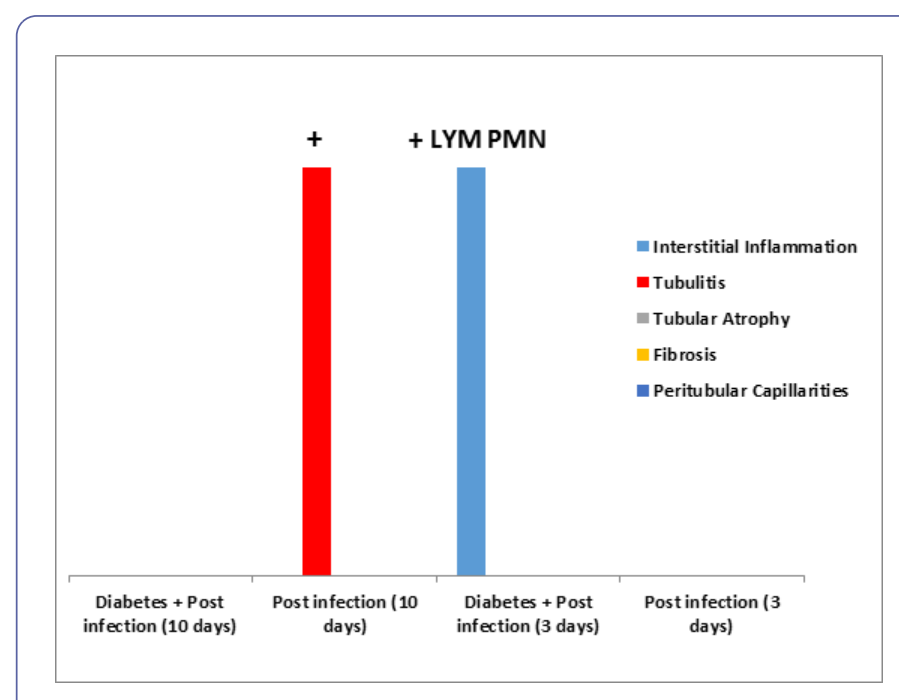

Figure 2: Tubulo-interstitial changes in post-infectious cases with and without diabetes.

The results of the immunofluorescence study were in concordance with the routine microscopy results and showed IgG and C3 positivity in Group IV at three and more extensively at ten days post induction of the infection (Table $\mathbf{3}$ and Figure $\mathbf{3}$ ). 


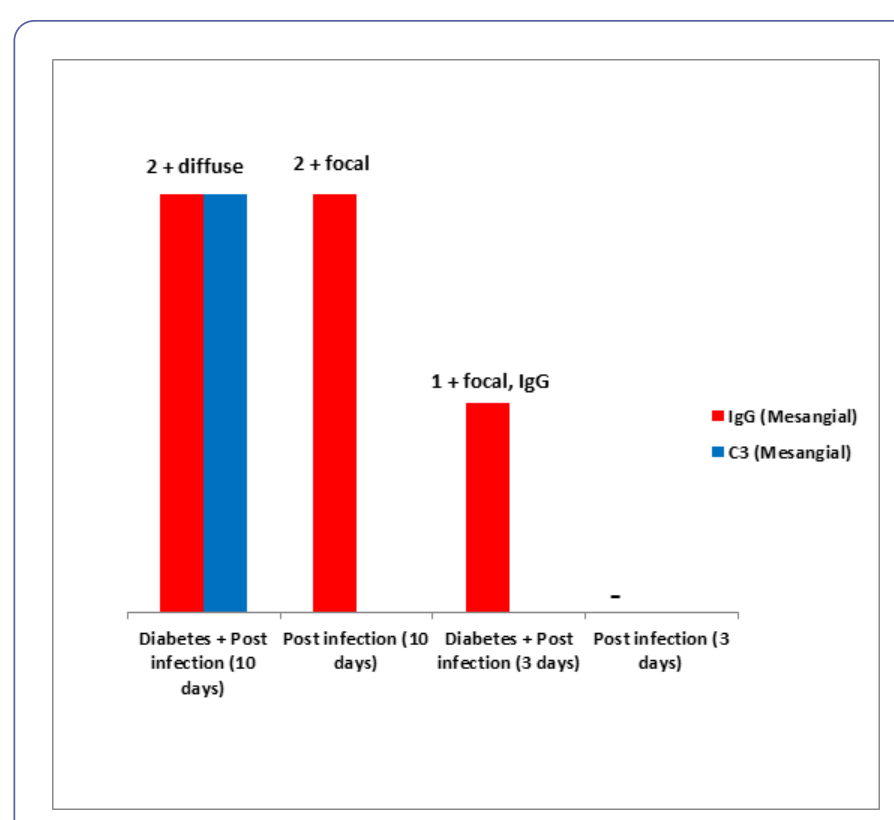

Figure 3: Immunofluorescence changes in post-infectious cases with and without diabetes.

The ultrastructural study showed immune dense deposition in the mesangial, paramesangial and subendothelial areas in group IV at ten days while at three days in the same group similar deposits were noted but without subendothelial dense deposits.

Table 3: Immunofluorescence findings in rats with and without diabetes at three and ten days post induction of acute infection.

\begin{tabular}{|l|l|l|l|}
\hline Immunofluorescence & IgG & C3 & \\
\hline & Mesangial & $\begin{array}{l}\text { Mesang } \\
\text { ial }\end{array}$ & $\begin{array}{c}\text { P- } \\
\text { value }\end{array}$ \\
\hline $\begin{array}{l}\text { Diabetes+ Post infection (10 days) } \\
(n=5 \text { rats) }\end{array}$ & $2+$ diffuse & $\begin{array}{l}2+ \\
\text { diffuse }\end{array}$ & 0.018 \\
\hline
\end{tabular}

\begin{tabular}{|l|l|l|l|}
\hline Post infection (10 days)( $\mathrm{n}=3$ rats $)$ & $2+$ focal & - & \\
\cline { 1 - 3 } $\begin{array}{l}\text { Diabetes+Post infection (3 days) }(\mathrm{n}=6 \\
\text { rats) }\end{array}$ & $1+$ focal, lgG & & \\
\hline Post infection (3 days)( $\mathrm{n}=2$ rats $)$ & & & \\
\hline
\end{tabular}

In group III mesangial and paramesangial dense deposits were noted at ten days and the amount of deposits was much less than in group IV at ten days while no dense deposits were noted at three days in group III.

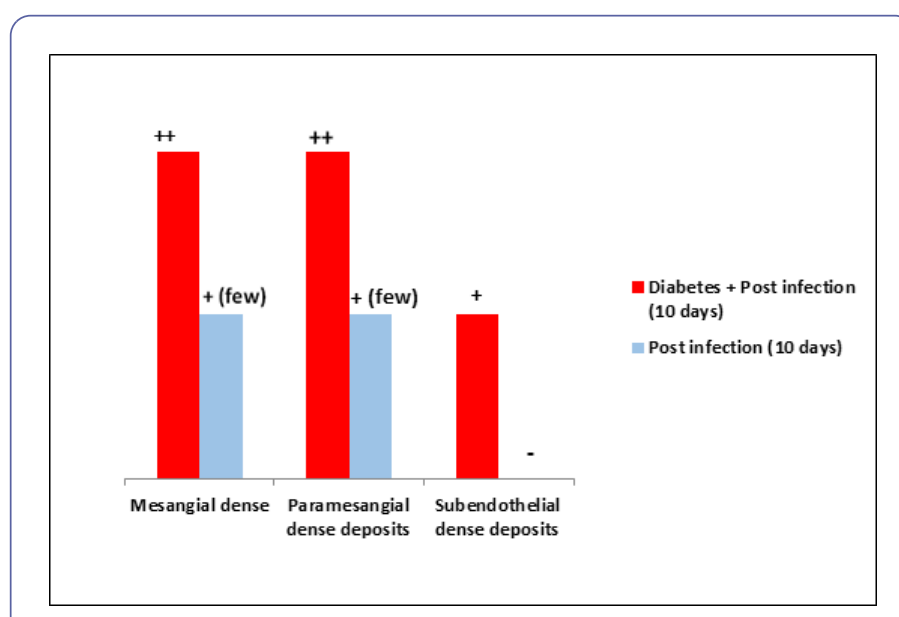

Figure 4: Electron microscopy changes in post-infectious cases with and without diabetes.

The thickness of the GBM was increased in groups II and IV and no statistical difference in the thickness was noted between the two groups. The effacement of the podocytes of the visceral epithelial cells was approximately $20 \%$ in groups II and IV and around $10 \%$ in the other groups (Table 4 and Figure 4 ).

Table 4: Ultrastructural findings in rats with and without diabetes at three and ten days post induction of acute infection.

\begin{tabular}{|c|c|c|c|c|}
\hline Electron microscopy & $\begin{array}{l}\text { Mesangial dense } \\
\text { deposits }\end{array}$ & $\begin{array}{l}\text { Paramesangial dense } \\
\text { deposits }\end{array}$ & $\begin{array}{l}\text { Subendothelial dense } \\
\text { deposits }\end{array}$ & P-value \\
\hline Diabetes + Post infection (10 days $)(n=5$ rats $)$ & ++ & ++ & + & 0.018 \\
\hline Post infection (10 days)( $n=3$ rats) & $+($ few $)$ & $+($ few $)$ & - & 0.018 \\
\hline
\end{tabular}

The serum creatinine was highest in group III while urinary protein was increased in group II. 


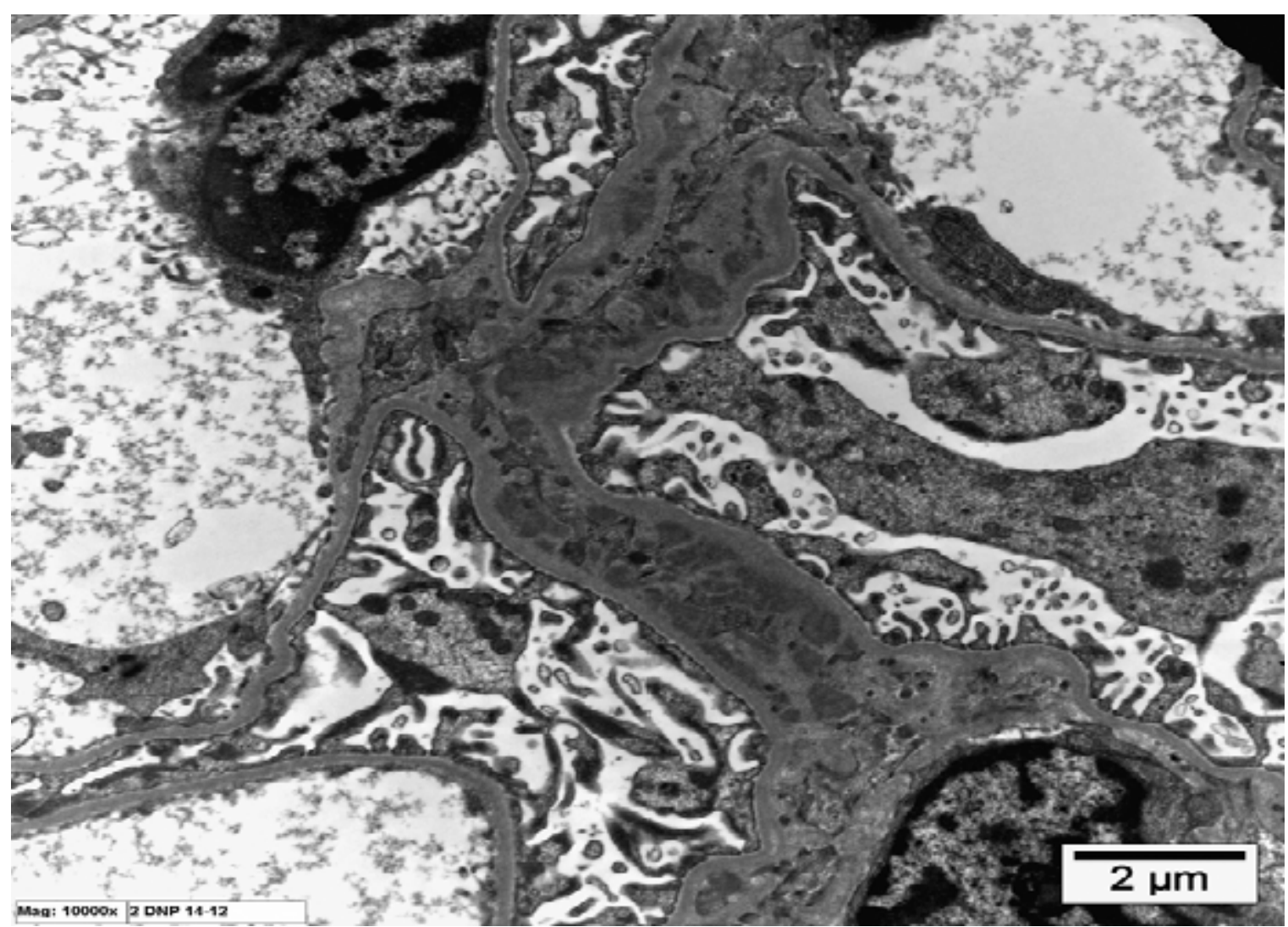

Figure 5: Electron photomicrograph of a kidney biopsy from a diabetic rat with APIGN showing the mesangial, paramesangial and subendothelial dense deposits. (Uranyl acetate, lead citrate X10000).

\section{Discussion}

The last few years unfolded many mysteries in renal pathology with better understanding of the pathogenetic mechanisms in a large number of non-neoplastic kidney diseases. One of the most important glomerular diseases in which our understanding of pathogenesis shed a new light on the pathological findings in kidney biopsy is diabetic nephropathy. The concurrence of diabetes and a nephritogenic infectious disease may have a tremendous impact on the kidney. The approach to the two concurrent diseases is based on the histological, the immunofluorescence findings and on the electron microscopy features. The advent of the molecular pathology and the different molecular events present in different diseases helped us to understand the outlines for the pattern of injury which affect the kidney. In this study, we tried to understand the pathogenesis and the pattern of injury that occur with infection in diabetic rats and compare it to rats with infection but without diabetes. Upon completion of the experiment, we noted that histopathological as well as the immunofluorescence and ultrastructural studies showed more prominent pathological changes with earlier onset of the features. Experimental models of infections in rabbits, rats, and mice with chemically induced diabetes have shown increased susceptibility to various bacterial and fungal infections [3,5-7]. Some studies to the contrary have shown no increased susceptibility to infection [8]. The present investigation was undertaken to determine the course of experimental APIGN in diabetic rats and to study the effects of diabetes on the course and severity of APIGN and the findings were in concordance with the literature in terms of the deleterious impact of diabetes on APIGN. The early stage of APIGN is known to show subendothelial, mesangial and paramesangial dense deposits (Figure 5), rather than subepithelial humps as it is shown in our experiment. The rats had to be sacrificed early (three and ten days post induction of the infection), as the diabetic status and the APIGN contributed in a drastic way to the loss of the animals, hence this measure was taken to avoid the death of the rats out of the working hours with all the side effects of autolysis.

Microbial populations of Candida albicans and Staphylococcus aureus were found to be higher and more persistent in diabetic rats $[9,10]$; in contrast, renal enterococcal populations were similarly sustained in both diabetic and control rats. Neither the duration of the diabetic state nor weight loss was found to be a contributing factor. Mechanisms responsible for the greater severity of renal infection in diabetes include defects in polymorphonuclear leukocyte function or cellular immunity. However, the earlier, more intense and sustained inflammatory response in the renal lesions in diabetic rats suggests that cell reactivity was not suppressed, but may have been relatively defective in clearing microorganisms from the kidney in this model, because of conditions in diabetes which favored urinary proliferation of microbes. 
The outcome of the study was very useful as it opened a new horizon in understanding the disease process and proved the theory that diabetic nephropathy induced a different pathological pattern in patients with concurrent APIG. The experiment gave the nephropathologist a further understanding of the two disease processes (diabetic nephropathy and APIG) and opened new horizon to a different therapeutic arsenal for patients with diabetic nephropathy and APIG, to treat them accordingly. It is well documented that diabetes may have a deleterious effect on patients when an infection occurs [11], however, in this study we stressed upon the aspect of APIGN in the setting of diabetes.

Although the sample size is limited due to expiry of the rat, however, the results appeared to be representative. Of note, the incidence of both diseases is prominent in our region, and the study outlined the adverse effects of diabetes on APIGN, in terms of onset and severity of the disease. The necessity of a better control of the glucose level may not only decrease the incidence of acute post-infectious glomerulonephritis but may also contribute to the development of a milder form of the disease.

\section{References}

1. Tancheva S, Valcheva-Kuzmanova SV, Radev RZ, Marinov MD, Boychev B, et al. (2011) A model of experimental acute hematogenous pyelonephritis in the rat. Folia Med 53: 63-68.

2. Philip JH, Jan J (1989) Weening. Relevance of experimental models for human nephropathology. Kidney Intern 35: 1015-1025.
3. Abdulla K Al- Hwiesh (2007) Xanthgranulomatous Pyelonephritis Associated with Staphylococcus Aureus. Saudi J Kidney Dis Transpl 18: 613-616.

4. Mazur E, Czerwińska E, Grochowalska A, Kozioł-Montewka M (2015) Concurrent peritonsillar abscess and poststreptococcal reactive arthritis complicating acute streptococcal tonsillitis in a young healthy adult: a case report. BMC Infect Dis 15: 50.

5. Cruickshank AH (1954) Resistance to infection in the alloxan B streptococcal infection. Infect Immun 39: 580- 585.

6. Forland M, Thomas V, Shelokov A (1977) Urinary tract infections in patients with diabetes mellitus. Studies on antibody coating of bacteria. JAMA 238: 1924-1926.

7. Obana Y, Nishino T, Tanino T (1987) Enhanced susceptibility of diabetic mice to gram-negative organisms. Nihon Saikingaku Zasshi 42: 653-661.

8. Edwards MS, Fuselier PA (1983) Enhanced susceptibility of mice with streptozotocin-induced diabetes to type II group activities of antimicrobial agents against Acinetobacter calcoaceticus. J Antimicrobial Chemother 15: 441-448.

9. Raffel L, Pitsakis P, Levison SP, Levison ME (1981) Experimental Candida albicans, Staphylococcus aureus, and Streptococcus faecalis pyelonephritis in diabetic rats. Infect Immun 34: 773-779.

10. Wang SY, Bu R, Zhang Q, Liang S, Wu J, et al. (2016) Clinical, Pathological, and Prognostic Characteristics of Glomerulonephritis Related to Staphylococcal Infection. Medicine 95: e3386.

11. Horace F, Gibson R, Williams N, Wharfe G, Nicholson A, et al. (2009) A woman with diabetes presenting with pyomyoma and treated with subtotal hysterectomy: a case report. J Med Case Reports 3: 7 . 